\title{
New Trends in Spinal Surgery: Less Invasive Anatomical Approach to the Spine. The Advantages of the Anterior Approach in Lumbar Spinal Fusion
}

\author{
Roberto BASSANI ${ }^{1}$, Carlotta MORSELLI ${ }^{1,2}$, Rosa BASCHIERA ${ }^{1}$, Stefano BROCK ${ }^{1}$, Dario GAVINO ${ }^{1}$, \\ Lorenzo PRANDONI ${ }^{3}$, Agostino CIRULLO $^{3}$, Laura MANGIAVINI ${ }^{1,4}$ \\ ${ }^{1}$ IRCCS Istituto Ortopedico Galeazzi, Milan, Italy \\ 2"Sapienza" - University, Department of Human Neuroscience, Rome, Italy \\ 3University of Milan, Residency Program in Orthopedics and Traumatology, Milan, Italy \\ ${ }^{4}$ University of Milan, Department of Biomedical Sciences for Health, Milan, Italy \\ Corresponding author: Carlotta MORSELLI carlotta.morselli@uniroma1.it
}

\section{ABSTRACT}

AIM: To describe the history of the anterior approach to the lumbar spine from the beginning to the minimal invasive techniques that the authors have developed, and to discuss its advantages and drawbacks.

MATERIAL and METHODS: The authors collected published data on the evolution of the anterior approach in the lumbar spine, and described its potential in different pathologies focusing on minimally invasive techniques.

RESULTS: Several successful techniques of anterior lumbar approach have been developed over the years, leading to a progressive less invasive anatomical exposure of the spine. Anterior approaches of the lumbar spine gained popularity as an alternative to posterior routes in the management of tumors, infections, traumas, degenerative or deformity diseases and as a salvage procedure after posterior surgery.

CONCLUSION: Advantages of the retroperitoneal anterior approach of the lumbar spine are well accepted: it preserves the anatomical structures of the abdomen and posterior tension band, avoiding muscle dissection. The implantation of lordotic cages with larger footprint improves local lordosis and fusion rate even in revision surgery. Drawbacks of traditional retroperitoneal approaches may be: vascular injury, deep venous thrombosis, risk of retrograde ejaculation in male in case of L5-S1 dissection. Therefore, several minimal invasive techniques have been developed to decrease the risks related to the traditional approaches. However, a long learning curve is required to achieve good skills and to manage possible technical concerns and complications.

KEYWORDS: Anterior lumbar interbody fusion, Video assisted, Lumbar revision surgery, Failed back syndrome, Complications

ABBREVIATIONS: ALIF: Anterior lumbar interbody fusion, IHP: Inferior hypogastric plexus, HD: High definition, ALL: Anterior longitudinal ligament, RE: Retrograde ejaculation, ASD: Adult spine deformity, QoL: Quality of life, SL: Segmental lordosis, TLIF: Transforaminal lumbar interbody fusion, LL: Lumbar lordosis, CT: Computed tomography, CTA: Computed tomography angiography, DVT: Deep venous thrombosis, LMWH: Low-molecular-weight heparins

Roberto BASSANI (1) : 0000-0003-4673-1020 Carlotta MORSELLI (1) : 0000-0003-1031-4874 Rosa BASCHIERA (i) : 0000-0002-7051-6024
Stefano BROCK

Dario GAVINO

(1) : $0000-0002-6682-5879$

Lorenzo PRANDONI (1) : 0000-0002-7428-0389
Agostino CIRULLO (1) : 0000-0002-4159-0946

Laura MANGIAVINI (1) : 0000-0003-1892-1249 


\section{INTRODUCTION}

A nterior lumbar interbody fusion (ALIF) is currently one of the most important approach to achieve interbody fusion for lumbar spinal disease among the available techniques $(3,5)$.

Degenerative conditions of the spine, severe flat-back (iatrogenic or degenerative), surgical revisions, and infections are the main indications of anterior lumbar surgery (5). The purpose of this review is to describe our experience in minimally invasive techniques, starting from the history of the anterior approach of the lumbar spine to the minimal invasive techniques we have developed.

\section{Evolution of the Anterior Approach}

In the early $19^{\text {th }}$ century pioneer surgeons, driven by a desire to find surgical solutions to treat Pott's disease, laid the foundations of anterior lumbar fusion surgery $(17,18,24)$.

In the 1932 Capener described for the first time a theoretical access to theanterior lumbar spine in a case of spondylolisthesis (10). Consequently, successful practical evolution of this approach was derived. The boost to develop anterior routes started as an alternative to posterior approaches in the management of tumors, infections, traumas, degenerative diseases, and as a salvage procedure when posterior grafting was inadvisable $(9,22,29)$. Burns described a tibial autologous bone graft inserted using a transperitoneal approach through L5 wedge for the first time in 1933 (9). Mercer performed the first interbody L5-S1 fusion with the same approach (22). Due to drawbacks and high surgical morbidity of open transperitoneal approaches (large skin incision, abdominal muscle trauma and major risk of retrograde ejaculation in male affected by L5-S1 dissection), less invasive routes have been explored, and ALIF technique gained new popularity in the 1980s (11).

Laparoscopic transperitoneal and mini-open retroperitoneal approaches have been then proposed to preserve the muscular integrity and function of the abdominal wall $(12,20,21,25)$. However, laparoscopic techniques presented several drawbacks related to loss of depth perception, the use of $\mathrm{CO}_{2}$ insufflation with several complications, and a long learning curve. Thus, this approach was soon abandoned $(12,20,25)$.

The anterior mini-open retroperitoneal exposure has acquired consensus as it causes less complications with better results. A muscle-sparing technique was optimized to reduce postoperative surgical morbidity, hospitalization, and rehabilitation time $(3,21)$.

Along with the development of less invasive anterior lumbar exposures, less invasive skin incisions have been described for single or multilevel procedures (3).

The classical median, paramedian or S-shaped skin incisions from the symphysis to the umbilicus have been progressively replaced by single transverse or pararectal shorter skin incisions in the lower third and the middle lower abdominal quadrant to expose retroperitoneal space (Figure 1) (3).
The anterior mini-open retroperitoneal approach requires a Pfannestiel skin incision less than $5 \mathrm{~cm}$ to expose the L5S1 space (Figure 1A). Alternatively, a pararectal skin incision on the left side is generally performed to expose the L4-L5 space. Left side is usually preferred due to easier mobilization of the left iliac vein and artery during dissection (from the right side common iliac vein or vena cava should be first mobilized especially for L4-L5 or higher spaces) (6). Multilevel ALIF exposures were traditionally performed using huge or multiple muscular and fascial incisions, with possible drawbacks, thus the need of suitable surgical incision has become crucial (3).

To reduce the invasiveness on muscular and fascial tissues, we recently proposed the original "keyhole" perinavel skin incision for a minimally invasive exposure of both single (i.e. L5-S1 or L4-L5 or L3-L4), and multiple levels (from L2-L3 to L5- S1), all in the retroperitoneal space (14) (Figure 1B, C).

We performed a $270^{\circ}$ perinavel incisionusing Colorado ${ }^{\circledR}$ microdissection needle (Figure 1B). The subcutaneous fatty tissue is then exposes with a semicircular dissection to produce a large fatty pad layer below the umbilicus, thus preserving superficial and deep vascular supply. The subdermal plexus (superficial blood supply) originates from the superficial superior and inferior epigastric arteries. The deeper vascular sources originate from the right and left deep superior and inferior epigastric vessels, the ligamentum teres hepaticum and the median umbilical ligament. Some perforating branches connect superficial and deep system, supplying the ventromedial skin of the lower area of the chest wall and the superior and periumbilical abdominal wall. Given this topographic vascular distribution, a $270^{\circ}$ skin incision (inverse horseshoe shaped skin incision) preserves vascular anastomosis saving for $90^{\circ}$ the superior part of the umbilicus; thus, it avoids umbilical necrosis (Figure 1).

To spare abdominal wall muscles, the anterior sheath of the left rectus abdominis muscle is longitudinally sectioned from the left side, about $2 \mathrm{~mm}$ lateral to the linea alba, and the left rectus muscle is retracted upward and laterally with careful blunt finger dissection of the extraperitoneal space. Once the rectus muscle is retracted, it is important to avoid tractions or lesions of the inferior epigastric vessels, which are located on the posterior side of the muscle. The peritoneal sac is then exposed and bluntly mobilized. The most lateral tract of the arcuate line (Douglas Line) is sectioned or bluntly dissected to expose the retroperitoneal space. The psoas muscle and genitofemoralis nerve are then visualized. During this step, the identification of the ureter, the left common iliac artery and vein is essential to localize and expose the L5-S1 disc (generally between iliac bifurcation), or the L4-L5 disc (laterally to the left common iliac vessels) (3). In multilevel ALIF procedures using the "keyhole" perinavel skin incision, we usually first approach the most inferior disc space and then we proceed cranially up to L2, if needed (Figure 1C).

In patients with high sacral slope (i.e high grade dysplastic spondylolisthesis), a less favorable inclination of the surgical field can cause an excessive traction of the cutaneous tissues; thus, careful dissection of each layer is mandatory to reduce tension. 


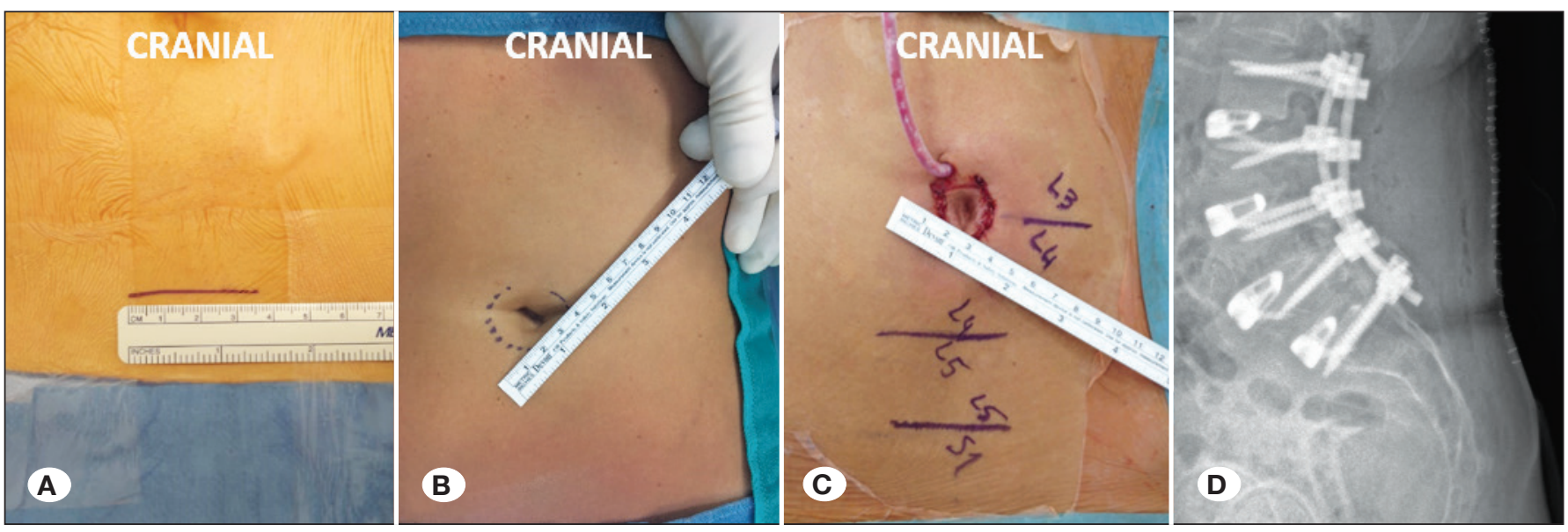

Figure 1: A) Standard mini-open skin incision. B, C) Modified Perinavel $270^{\circ}$ skin incision sec. Bassani used for multilevel disc approach. D) Post-operative lateral X-Ray showing multilevel ALIF implant performed with perinavel skin incision.

Once the L5-S1 disc is exposed, the common iliac vessels are retracted and protected; finally the middle sacral vessels are ligated to avoid injury to the inferior hypogastric plexus (IHP). Coagulation with bipolar forceps could produce thermal or electrical injury to IHP. The utilization of the monopolar knife must be avoided.

Once vessels are mobilized and complete exposure of the disc is performed, hemostatic agents with oxidized cellulose $\left(\right.$ Surgicel ${ }^{\circledR}$ ) are placed behind each retractor blade to protect the veins. The retracting blades are generally fixed to the vertebral body with dedicated pins. This very stable configuration avoids the risk of soft tissue or vascular injury due to retractor's accidental movements during discectomy or cage implant. An autostable ring is then placed to connect the handles of the retracting blades to obtain a 360 degree stability and a complete view of the surgical field.

More vertical and favorable surgical corridor is encountered above L5. Aorta, cava and common iliac veins and arteries are retracted from the left to the right side towards the midline. To avoid traction or tears, ligature of the ileolumbar veinis often necessary.

Subsequently, aorta is medially displaced to expose the lateral aspect of the L3-L4 space. Segmental vessels from the aorta and vena cava on the anterolateral aspect of the vertebral body should be carefully identified and ligated.

In our series, using perinavel approach, postoperative complications' rate related to skin incision was $4.12 \%$ (3), and all the complications were conservatively managed. The rate of venous injury with the single "keyhole" perinavel incision was similar to the current literature (3.09\% vs. 3.07\%), demonstrating its safety compared to traditional approaches (6). A single perinavel incision, acting as a "sliding door" to the retroperitoneal space, allows for a better exposure of the anterior midline of the lumbar spine. Moreover, this technique reduces postoperative abdominal pain, abdominal morbidity, and blood loss allowing for a shorter postoperative recovery, bed rest and length of hospitalization if compared to other incisions.

\section{Further Innovative Techniques}

In the last decades, autologous bone grafts used as "spacer" and fusion devices have been replaced by other special tools with different footprints, size and lordotic shapes (titanium, peek, tantalum, and other allograft cages). Dedicated deeper autostable retractors with special blades have been designed to obtain optimal visualization of the surgical field. To reduce operative time and complications, we usually recommend the use of video assistance to perform better disc preparation and cage implantation (Figure 2) (4)

Once anterior interbody surface has been prepared and the autostable retractor fixed, a rigid endoscope $\left[10 \mathrm{~mm}, 30^{\circ}\right.$, cold light endoscope coupled to a High Definition (HD) screen] allows for a better view of all phases of disc preparation both in single and multilevel approaches. The anterior longitudinal ligament (ALL) is then sectioned to create two lateral flaps protecting the surgical corridor. After a complete discectomy, the deep light source allows for a complete endplates visualization, until the posterior annulus. A complete and accurate discectomy is one of the most important step to obtain the fusion (Figure 3A-F). Furthermore, the HD screen visualization permits a better coordination and quicker collaboration among staff components (assistants, scrub nurses), reducing operation time (4).

In our experience, the video assisted mini-open retroperitoneal anterior approach does not significantly increase the access-related complications' rate compared to the current literature (4). On 269 patients, we registered $1.1 \%$ of vascular complication (venous), and $2.75 \%$ of retrograde ejaculation in males; whereas in the literature vascular complication rate ranges from $1.9 \%$ to $18 \%$, retrograde ejaculation from 0.44 to $5 \%$ in standard mini-open approaches $(7,15,16,27,28)$.

\section{ALIF in Degenerative Lumbar Spinal Disease}

Despite the initial higher drawbacks of the anterior approach compared to potential benefits, the subsequent progressions to safer and advanced techniques have increased ALIF usefulness as an alternative procedure to standard posterior 

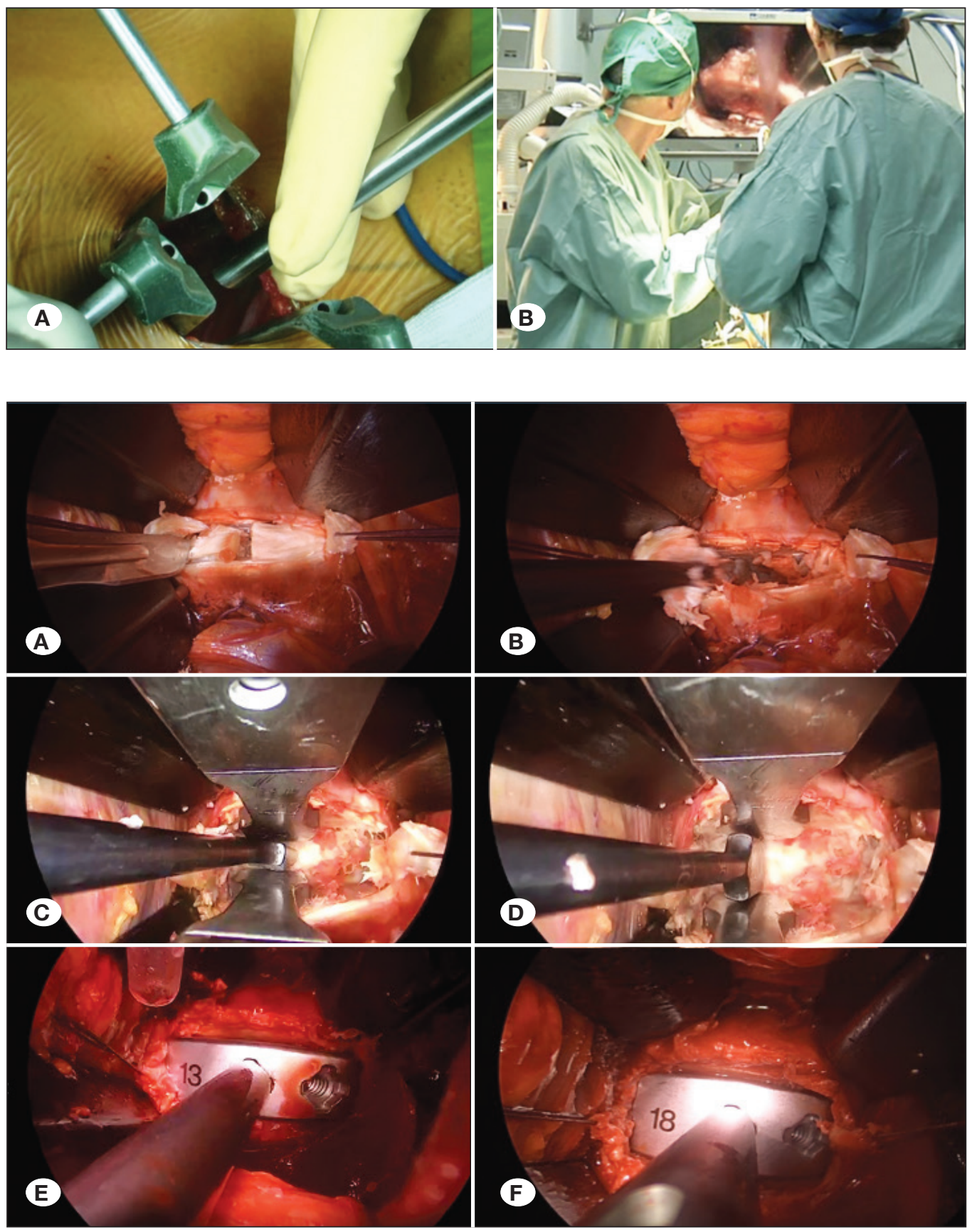

Figure 2: A) High Definition endoscopic assistance $\left(30^{\circ}\right)$ introduction. B) Videoassisted ALIF procedure and operative theatre set-up.
Figure 3: A) Intervertebral L5-S1 disc incision and "Window exposure". B) L5-S1 discectomy. C, D) Opening of the disc space with spreader. E, F) Increased size templates implanted to find the proper fit. approaches, especially in case of degenerative diseases $(1,3,11)$. Mechanical low back pain can origin from disc degeneration or facet joints' arthritis. Disc degeneration due to nucleus progressive dehydration and recurring annulus injuries can lead to a symptomatic progressive disc height reduction, and ultimately to complete collapse of the disc. Facet joint arthritis, ligamentum flavum hypertrophy, and the concomitant osteophytes in presence of posterior tension band weakness can cause degenerative instability with secondary canal stenosis.
Spinal interbody fusion (IF) has been widely recognized to improve clinical outcome in surgical treatment of degenerative pathology of the lumbar spine (5).

ALIF is actually employed in the surgical treatment of degenerative disc disease (DDD), in spondylolisthesis (both degenerative and isthmic), or to achieve fusion in recurrent lumbar disc herniation and post-discectomy kyphosis with good results even in terms of proper lumbar lordosis restoration (Figure 4-6) (3,5). 


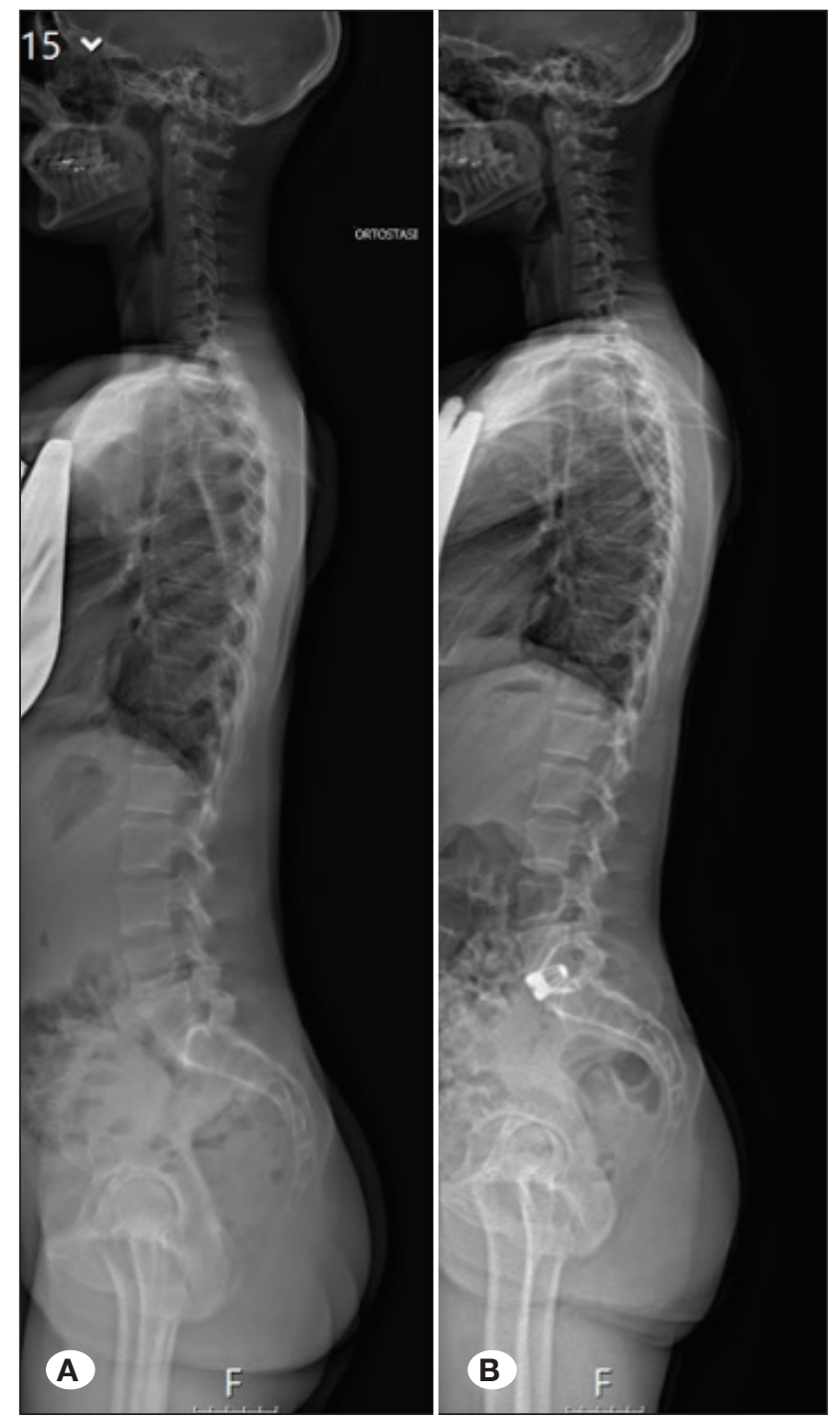

Figure 4: A) Pre and B) post-operative full standing spine $X$-RaysEOS of a L5-S1 ALIF with lordotic cage for treatment of DDD.
According to our experience in the management of a single level L5-S1 DDD, ALIF showed better results compared to trans foraminal interbody fusion (TLIF) in terms of surgical (lower blood loss and shorter surgical time), radiological (SL improving) and clinical (early postoperative pain reduction) outcomes (5).

In the treatment of DDD as well as in recurrent lumbar disc herniation and post-discectomy kyphosis ALIF approach avoids muscle denervation and fat degeneration (5). Furthermore, the fusion rate can be increased by placing the cage anteriorly, in the bony surface area that supports about the $80 \%$ of axial load in the upright standing position. In the management of degenerative spondylolisthesis, ALIF corrects disc height and it stabilizes the anterior column. Compared to posterior IF, a direct anterior approach to the disc allows for restoring an optimal segmental lordosis (SL) as well as an open foraminal spaces, leading to an indirect decompression of the nerve roots (5).

\section{ALIF in the Correction of Sagittal Alignment and Revision Surgery}

Anterior approach gained further popularity among spine surgeons in the treatment of complex spinal disease like adult spine deformity (ASD), and revision surgery (Figure 7, 8) $(1,5)$, due to the development of lordotic and hyperlordotic shaped cages. The goal of a complex correction of a spinal deformity in adulthood and of a revision surgery is to improve the quality of life (QoL), achieving sagittal and coronal imbalance correction to get a stable spinal fusion, and pain relief. Several studies demonstrated that the loss of lumbar lordosis (LL) can lead to sagittal malalignment with QoL impairment (1). Therefore, ideal lumbar lordosis restoration and the bony fusion represent the most important aims of the corrective surgery.

Traditionally, cages used in anterior approach have between 8 and 12 degrees of lordosis (2). Recently, multilevel cages (with lordosis from $15^{\circ}$ to $30^{\circ}$ ) have been adopted to correct sagittal malalignment with significant improvement of SL instead of huge posterior pedicle subtraction osteotomies (2).
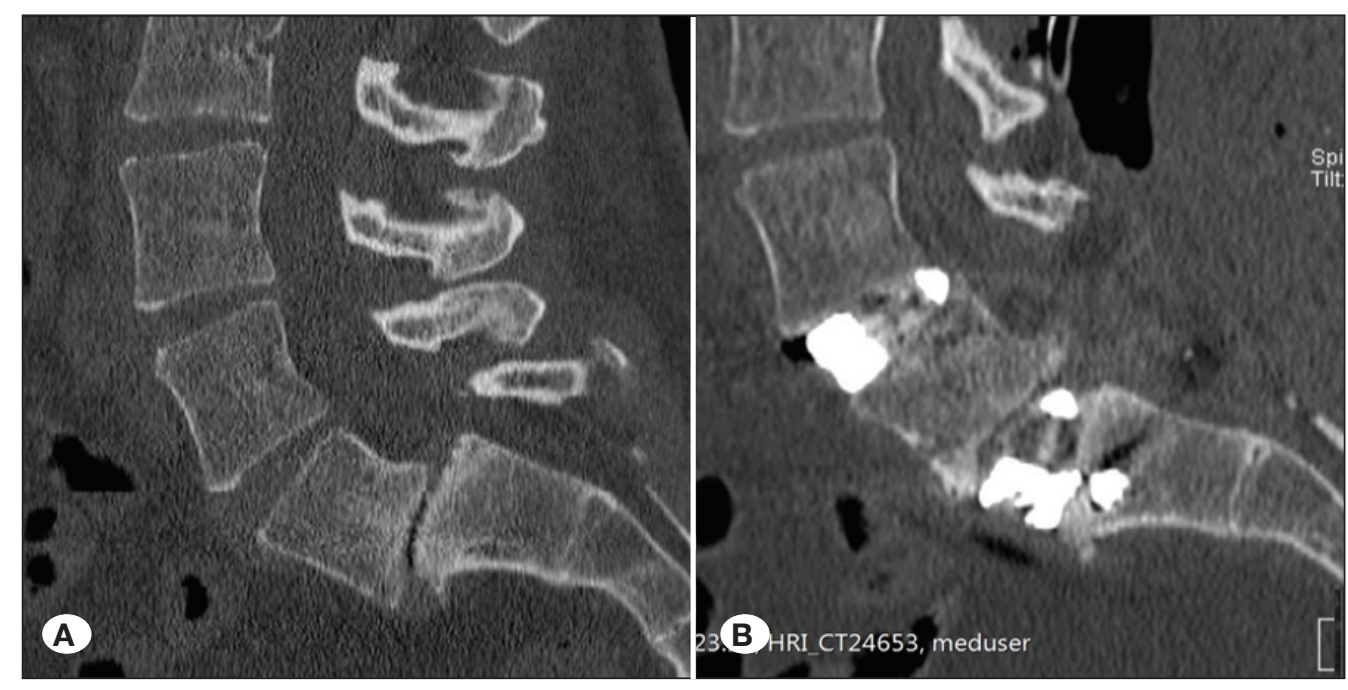

Figure 5: A) Pre and B) postoperative CT-Scan of a L4L5-S1 ALIF for treatment of $2^{\circ}$ Spondylolisthesis. 

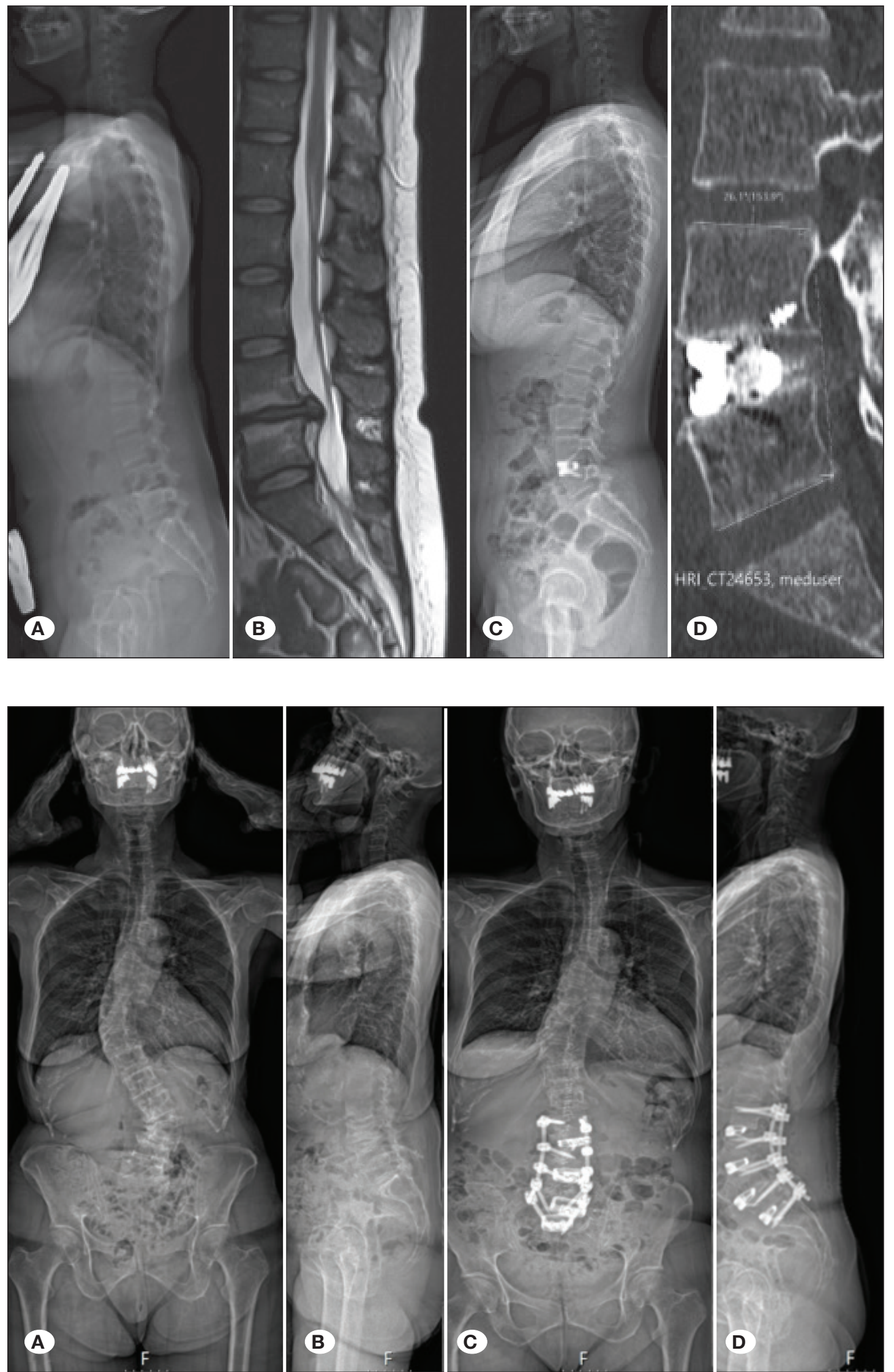
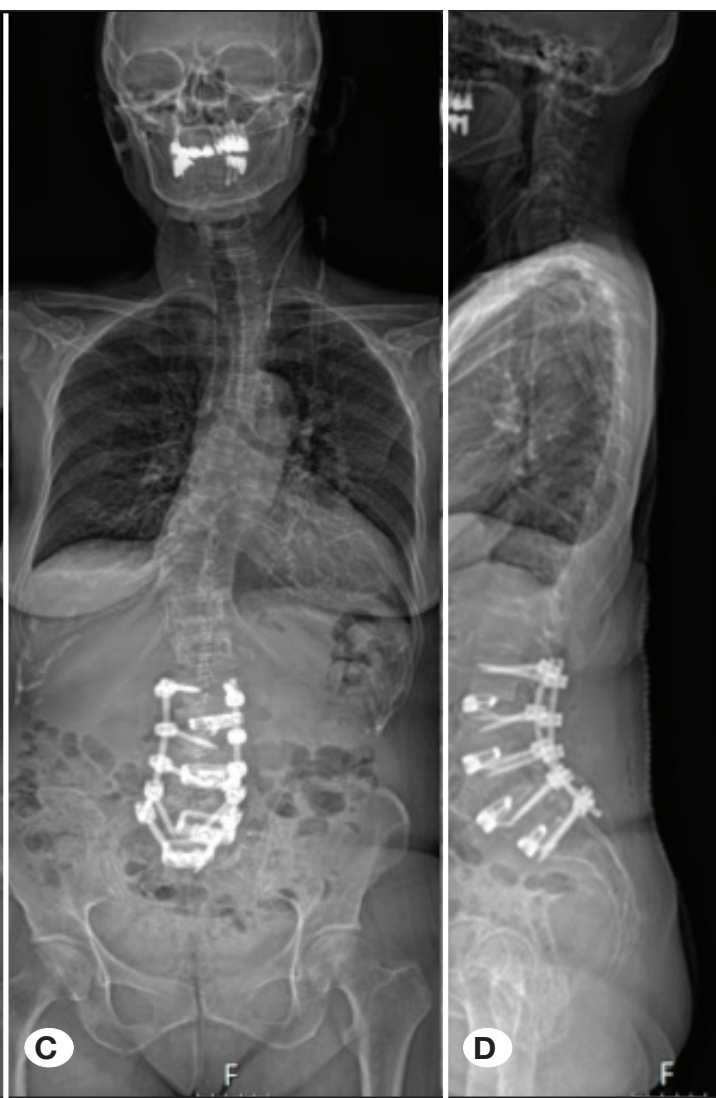

Figure 7: A, B) Pre and C, D) post-operative full standing spine X-Rays-EOS of a multilevel ALIF for treatment of adult deformity scoliosis. 

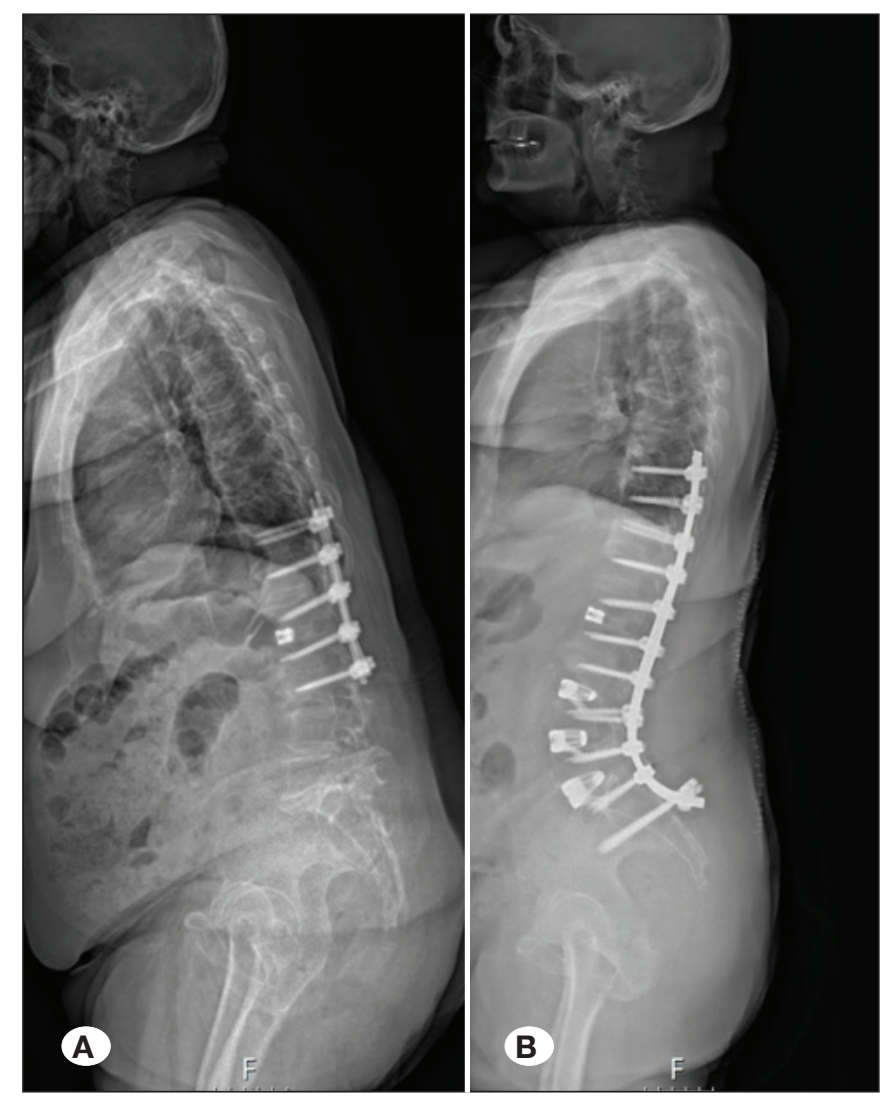

Figure 8: A) Pre and B) post-operative full standing spine X-RaysEOS of a multilevel ALIF for balance restoration in revision surgery.

ALIF safety and efficacy in ASD surgery have been well documented in the treatment of pain due to sagittal imbalance $(1,5)$, and in residual hypolordosis after failed posterior fusion $(13,23)$.

The ALL resection with an anterior surgical approach allows for a direct vision to the disc. After a complete discectomy and posterior annulus release, a powerful interbody distraction permits an optimal disc height restoration and foraminal decompression. The implantation of a large and lordotic cage provides great primary stability, increasing potential fusion rate and restoring SL (Figure 4) (2,5). In presence of posterior hardware, a strong anterior support permits to overpower posterior instrumentation avoiding osteotomies and reducing surgical time and perioperative blood loss (Figure 9) (13).

The capability of ALIF to restore SL and to correct sagittal imbalance has been widely described in the literature during the years (5). Many studies have demonstrated the superiority of ALIF compared to TLIF in terms of disc height and LL restoration (5).

ALIF procedure in ASD surgery is particularly indicated in presence of loss of LL between L4-S1 (22). The ideal proportion of LL increased gradually, from $4 \%$ for L1-L2 to $35 \%$ for L5-S1 (2). Double or multilevel ALIF offers an harmonic and graduated correction of the LL avoiding injury

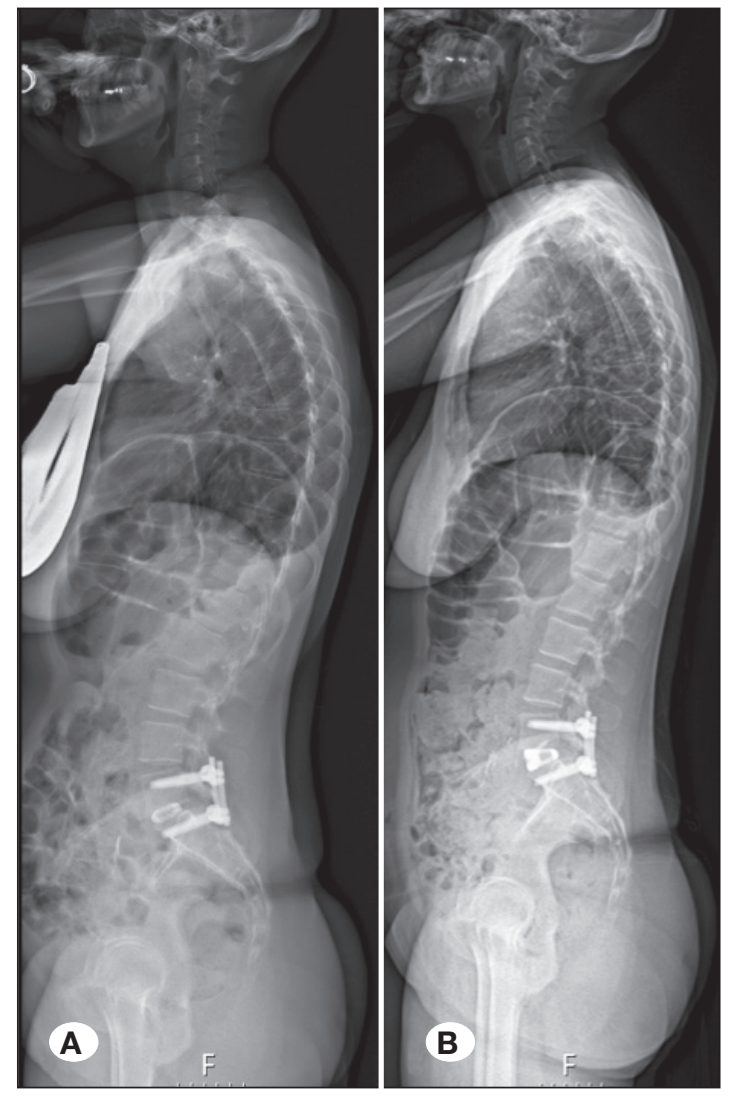

Figure 9: A) Pre and B) post-operative full standing spine X-RaysEOS of a L5-S1 ALIF for anterior cage removal and restoration of sagittal balance.

of the posterior muscle tension band (1). Thus, the perinavel approach, working as a "sliding door", allow for performing single stage multilevel ALIF from L2 to S1 with a lower rate of complications (6).

ALIF procedure achieves SL restoration with lower complications and higher fusion rates compared to posterior osteotomies (13). These advantages should be carefully considered in the planning of ASD or revision surgery.

The anterior exposure of lumbar discs can also be useful in the revision surgery of unsuccessful posterior interbody fusion due to loss of SL, cage nonunions, mobilization or infection. Posterior approach can cause perineural scarring tissues, thus it may increase the risk of dural tear, nerve root injury or infections. Anterior naive approach to the disc can limit these complications with a powerful correction of local kyphosis $(3,23)$.

\section{ALIF Complications and Their Management}

Despite the recent ALIF popularity among spinal surgeons, the potential risk to injure retroperitoneal or intra-peritoneal structures remains consistent; therefore, its application requires a long learning curve (27).

Complication rate of ALIF procedure has been highly variable, accounting between 8.4 to $31.1 \%$ in the large series 
depending on differences in complication endpoints reported in the studies and on heterogeneity of surgeries (i.e., singlelevel or multilevel ALIF) $(14,26)$.

The main concerns of this approach are vascular injuries and retrograde ejaculation $(\mathrm{RE})(7,8,15,19,26)$.

Intraoperative venous injuries (usually left iliac vein), occurs between 1.9 to $18 \%$ in large series, and mostly occurs during L4-L5 exposure (iliolumbar vein tear) (7).

To avoid injuries during disc exposure, we recommend a careful mobilization of the artery and vein starting as distally as possible without mechanical stresses. In case of spondylolisthesis or anterior revision surgery, careful removal of all fibrous tissue between the anterior spinal surface and veins is crucial. The iliolumbar vein or L4 segmental vessels should be always identified, mobilized and often ligated to avoid tears at the junction to the iliac vein, thus facilitating anterior exposure of L4-L5 disc space.

If vein injury occurs during exposure, early identification and bleeding control should be promptly performed with vein compression or suture repair. In cases of small tears $(<3 \mathrm{~mm})$, compression and hemostatic agents generally can control more than $90 \%$ of the bleeding and suturing may not be required. In larger venous injuries, a 5-0 prolene suture (placed in eight-fashion) or vascular clips should be applied.

Intraoperative positioning of pulse oxymeter on the left great toe may be useful in case of protracted retraction of the vessels to avoid ischemic complications, especially in documented calcified iliac arteries.

Intraoperative arterial bleeding, a very rare complication, can be managed with direct suture repair.

Early postoperative vascular complications can be due to active bleeding, generally originating from inferior epigastric vessels injured by retractors during the exposure. Although superficial hematoma can be conservatively treated, a retroperitoneal hematoma should be carefully investigated with computed tomography angiography (CTA), and CT scanning. Bleeding from arterial rectus sheath supply can occur in the early postoperative time (12-24 hours). It is a rare but potentially life-threatening complication, presenting with abdominal or back pain, evidence of a palpable abdominal wall mass and symptoms and signs of hypovolemic shock.

Iliac or deep venous thrombosis (DVP) as well as arterial injuries are unusual (occur from $0.45 \%$ to $1 \%$ ) and they are associated with prolonged vessels retraction or manipulation. Postoperative use of thrombosis prophylaxis agents (i.e lowmolecular-weight heparins) should be advocated in cases of thrombotic occlusion.

A ureteral injury during first exposure is uncommon but possible. Risk of venous and ureteral injuries increases in patients who underwent previous anterior retroperitoneal approach $(23,26)$. As the ureter may be enceased in scar tissue, we place a ureteral stent in all our revision operations to prevent further injury.

A related complication in males is represented by RE. Symptoms duration could be transient or permanent with conse- quent impact on fertility rates (8). Surgical technique (blunt dissection; avoiding monopolar coagulation), and surgeon experience, may influence RE rate that ranges from $0 \%$ to $4.1 \%$ in retroperitoneal approach up to $13.3 \%$ in patients undergoing transperitoneal approach (8). Perivascular fibrous tissue containing the sympathetic fibers of the hypogastric plexus is adherent to the posterior surface of the peritoneum and laying on the anterior surface of the lumbosacral spine. To complete disc exposure in L5-S1 sacral vessels should be ligated and coagulated with potential injury of IHP. To preserve the function of hypogastric plexus, the use of bipolar electrocautery is mandatory only on well identified small vessels to avoid electrical and/or thermal injuries.

Despite these complications, innovative techniques have decreased the complications' rate; however, the potential surgical risks of anterior lumbar exposure require advanced technical skills (19). Thus, some authors recommended access surgeon assistance although to our knowledge, no consistent results are reported in the literature (26).

In our practice, orthopedic spine surgeons or neurosurgeons with expertise on anterior approaches perform the anterior exposure by themselves. According to other results in literature, we reported a low rate of perioperative access related complications $(3.1 \%)(4,5,19,26)$.

\section{CONCLUSION}

ALIF advantages are well accepted and useful for degenerative disorders, spinal deformities and revision cases. Implantation of huge and lordotic cages improves fusion rate, thus restoring proper sagittal lumbar profile with preservation of the posterior muscles and bleeding reduction. Surgical time and recovery are faster. The related potential risks remain consistent without experience. The ability to manage surgical complications increases with experience. An adequate progressive learning curve may train spine surgeons to gradually and safely perform the exposure. Knowledge of abdominal and vascular anatomy is mandatory to prevent potential complications.

\section{- REFERENCES}

1. Ahern DP, Welch-Phillips A, Cawley DT, Butler JS: Is multilevel anterior lumbar interbody fusion (ALIF) superior to pedicle subtraction osteotomy (PSO) for degenerative lumbar deformity? Clin Spine Surg 33(1):1-4, 2020

2. Bassani R, Gregori F, Morselli C, Querenghi A, Giacalone A, Agnoletto M, Mangiavini L, Peretti GM: Use of lordotic cages in L5-S1 anterior lumbar interbody fusion (ALIF) procedures. J Biol Regul Homeost Agents 34(4 Suppl 3):23-28, 2020

3. Bassani R, Gregori F, Peretti G: Evolution of the anterior approach in lumbar spine fusion. World Neurosurg 131:391398, 2019

4. Bassani R, Morselli C, Querenghi AM, Agnoletto M, Brambilla L, Mangiavini L: Video-assisted anterior retroperitoneal approach to the lumbar spine. A minimally invasive technique improved by the use of an endoscopic camera to treat lumbar spinal diseases. Consideration over 269 patients. J Biol Regul Homeost Agents 34(4 Suppl 3):7-14, 2020 
5. Bassani R, Morselli C, Querenghi AM, Nuara A, Sconfienza LM, Peretti GM: Functional and radiological outcome of anterior retroperitoneal versus posterior transforaminal interbody fusion in the management of single-level lumbar degenerative disease. Neurosurg Focus 49(3):E2, 2020

6. Bassani R, Querenghi AM, Cecchinato R, Morselli C, Casero G, Gavino D, Brock S, Ferlinghetti C: A new "keyhole" approach for multilevel anterior lumbar interbody fusion: The perinavel approach. Technical note and literature review. Eur Spine J 27:1956-1963, 2018

7. Brau SA, Delamarter RB, Schiffman ML, Williams LA, Watkins RG: Vascular injury during anterior lumbar surgery. Spine $J$ 4: 409-412, 2004

8. Burkus JK, Dryer RF, Peloza JH: Retrograde ejaculation following single-level anterior lumbar surgery with or without recombinant human bone morphogenetic protein-2 in 5 randomized controlled trials: Clinical article. J Neurosurg Spine 18(2):112-121, 2013

9. Burns $\mathrm{BH}$ : An operation for spondylolisthesis. Lancet 221:1233, 1933

10. Capener N: Spondylolisthesis. Br J Surg 19:374-386, 1932

11. Chow SP, Leong JC, Ma A, Yau AC: Anterior spinal fusion for deranged lumbar intervertebral disc. Spine (Phila Pa 1976) 5:452-458, 1980

12. Cowles RA, Taheri PA, Sweeney JF, Graziano GP: Efficacy of the laparoscopic approach for anterior lumbar spinal fusion. Surgery 128:589-596, 2000

13. Formica M, Quarto E, Zanirato A, Mosconi L, LontaroBaracchini M, Alessio-Mazzola M, Felli L: ALIF in the correction of spinal sagittal misalignment. A systematic review of literature. Eur Spine J 30(1):50-62, 2021

14. Giang G, Mobbs R, Phan S, Tran TM, Phan K: Evaluating outcomes of stand-alone anterior lumbar interbody fusion: A systematic review. World Neurosurg 104:259-271, 2017

15. Ikard RW: Methods and complications of anterior exposure of the thoracic and lumbar spine. Arch Surg 141:1025-1034, 2006

16. Inamasu J, Guiot BH: Vascular injury and complication in neurosurgical spine surgery. Acta Neurochir 148:375-387, 2006
17. Ito $\mathrm{H}$, Asami G: Lumbosacral sympathetic ganglionectomy. Am J Surg 15(1):26-38, 1932

18. Ito H, Tsuchiya J, Asami G: A new radical operation for Pott's disease. J Bone Jt Surg 16(3):499-515, 1934

19. Jarrett CD, Heller JG, Tsai L: Anterior exposure of the lumbar spine with and without an "access surgeon". Morbidity analysis of 265 consecutive cases. J Spinal Disord Tech 22(8): 559-564, 2009

20. Mahvi DM, Zdeblick TA: A prospective study of laparoscopic spinal fusion: Technique and operative complications. Ann Surg 224(1):85-90, 1996

21. Mayer HM: A new microsurgical technique for minimally invasive anterior lumbar interbody fusion. Spine (Phila Pa 1976) 22:691-699, 1997

22. Mercer W: Spondylolisthesis with a description of a new method of operative treatment and notes of ten cases. Edinb Med J 43:545-572, 1936

23. Mobbs RJ, Phan K, Thayaparan GK, Rao PJ: Anterior lumbar interbody fusion as a salvage technique for pseudoarthrosis following posterior lumbar fusion surgery. Glob Spine J 6:1420, 2016

24. Müller W: Transperitoneale freilegung der wirbelsaule bei tuberkuloser spondylitis. Dtsch Ztschr Chir 85:128, 1906

25. Obenchain TG: Laparoscopic lumbar discectomy: Case report. J Laparoendosc Surg 1:145-149, 1991

26. Phan K, Xu J, Scherman DB, Rao PJ, Mobbs RJ: Anterior lumbar interbody fusion with and without an "access surgeon": A systematic review and meta-analysis. Spine 42(10):E592-E601 2017

27. Quraishi NA, Konig M, Booker SJ, Shafafy M, Boszczyk BM, Grevitt MP, Mehdian H, Webb JK: Access related complications in anterior lumbar surgery performed by spinal surgeons. Eur Spine J 22 Suppl 1:S16-20, 2013

28. Sclafani JA, Kim CW: Complications associated with the initial learning curve of minimally invasive spine surgery a systematic review. Clin Orthop Relat Res 472(6):1711-1717, 2014

29. Stauffer RN, Coventry MB: Anterior interbody lumbar spine fusion. Analysis of Mayo Clinic series. J Bone Joint Surg Am 54:756-768, 1972 